\title{
A K-WISHART MARKOV RANDOM FIELD MODEL FOR CLUSTERING OF POLARIMETRIC SAR IMAGERY
}

\author{
V. Akbari *, G. Moser **, A. P. Doulgeris *, S. N. Anfinsen *, T. Eltoft *, and S. B. Serpico ** \\ * Department of Physics and Technology, University of Troms $\phi$, NO-9037, Troms $\phi$, Norway \\ ** Department of Biophysical and Electronic Engineering, University of Genoa, IT-16145, Genoa, Italy
}

\begin{abstract}
A clustering method that combines an advanced statistical distribution with spatial contextual information is proposed for multilook polarimetric synthetic aperture radar (PolSAR) data. It is based on a Markov random field (MRF) model that integrates a K-Wishart distribution for the PolSAR data statistics conditioned to each image cluster and a Potts model for the spatial context. Specifically, the proposed algorithm is constructed based upon the expectation maximization (EM) algorithm. A new formulation of EM is developed to jointly address parameter estimation in the K-Wishart distribution and the spatial context model, and also minimization of the energy function. Experiments are presented with simulated and real quad-pol L-band data.
\end{abstract}

Key words: Polarimetric synthetic aperture radar (PolSAR); expectation-maximization (EM); K-Wishart; Markov Random Field (MRF); statistical modeling.

\section{INTRODUCTION}

Polarimetric SAR (PolSAR) data are complex multidimensional image data, which can be analyzed adopting several processing schemes. In the literature, we find that much emphasis has been put on analysis based on target decomposition theorems. Through this approach, information about scattering mechanisms can be gained. The knowledge of the exact statistical properties of PolSAR data founds the basis for another strategy of multidimensional image analysis, which in some cases is complementary to the target decomposition approach.

Analysis of real SAR images often reveals that nonGaussian models give better representation of the data, compared to complex Gaussian distributions, implying that processing algorithms based on non-Gaussian statistics should improve performance. The doubly stochastic product model has been widely used in non-Gaussian modeling, processing, and analysis of single- and multiPolSAR images. The model states that, under certain conditions, the complex-valued scattering vector results as the product of two independent random variables: a circular complex multinormal speckle noise component and a real scalar texture component. Several distributions could be used to model SAR image texture with different spatial correlation properties and various degrees of inhomogeneity $[1,2]$. Statistical properties are widely used for image segmentation and land cover classification of PolSAR data (e.g., [1]).

This paper addresses the problem of contextual polarimetric SAR image clustering by combining advanced statistical modeling and MRFs. Polarimetric SAR image segmentation is commonly performed with the Gaussianbased Wishart clustering algorithm operating on a pixelby-pixel basis [3]. In this study, we use the more advanced non-Gaussian K-Wishart clustering algorithm [1], that additionally accounts for potential textural differences in the classes, to represent the individual pixelwise statistical properties, and integrate contextual information of the associated image data in the analysis process by MRF modeling [4]. MRFs represent a general family of stochastic models that allow both contextual information and further information sources [4] to be integrated in Bayesian image processing by formulating the maximum a-posteriori (MAP) decision rule as the minimization of suitable energy functions. MRF models have been used in remote-sensing to address many image-analysis problems, including (supervised and unsupervised) classification, segmentation, texture extraction, denoising, and change detection (see, e.g., $[4,5,6]$ ).

The proposed contextual clustering method uses a Markovian energy function that integrates a K-Wishart model for the PolSAR data statistics conditioned to each image cluster and an MRF model for the spatial context. Specifically, the proposed algorithm is constructed based upon the expectation maximization (EM) algorithm [7]. EM is an iterative parameter estimation technique, developed for parametric-modeling problems characterized by data incompleteness and converging to a local, at least, maximum of the log-likelihood function [7, 8]. Here, a novel formulation of EM is developed to jointly address both parameter estimation and minimization of the energy function. The resulting algorithm works in an iterative manner where, in each iteration, the current cluster parameter estimates are used to segment the image, and the new segments are used to recompute the cluster parameters. 


\section{PIXEL-WISE STATISTICAL ANALYSIS}

After multi-looking, each pixel is represented by $\mathbf{C}$, the multi-look complex (MLC) covariance matrix of the scattering vector. The non-Gaussian product model describes $\mathbf{C}$ as the product of a texture term and a Wishart distributed speckle term [2]. Assuming that the texture has higher spatial correlation than the speckle over small local neighborhoods, the doubly stochastic product model for multilook PolSAR data is given by:

$$
\mathbf{C}=T \mathbf{W} ; \quad \mathbf{W} \sim \mathcal{W}_{d}^{\mathbb{C}}(L, \boldsymbol{\Sigma}) .
$$

where $d$ is the dimension of the scattering vector, $T$ and $\mathbf{W}$ model texture and speckle, respectively and $\mathcal{W}_{d}^{\mathbb{C}}(L, \boldsymbol{\Sigma})$ denotes the scaled complex Wishart distribution with parameters $L$, the number of looks, and $\boldsymbol{\Sigma}=\mathbf{E}\{\mathbf{C}\}$ [10]. The non-Gaussian nature of the product model depends on the specific model for the scalar texture variable $T$ [2].

\subsection{K-Wishart distribution}

If the texture term of the product model is given by the gamma distribution with probability density function (PDF) given by

$$
p_{T}(t ; \mu, \alpha)=\left(\frac{\alpha}{\mu}\right)^{\alpha} \frac{t^{\alpha-1}}{\Gamma(\alpha)} \exp \left(-\frac{\alpha}{\mu} t\right),
$$

with shape parameter $\alpha>0$ and unit mean value $\mu=$ $\mathbf{E}\{T\}=1$. Then the marginal distribution for $\mathrm{C}$ may be obtained by integrating the conditional PDF over the prior distribution of $T$, that is

$p_{C}(\mathbf{C})=\int_{0}^{\infty} p_{C \mid T}(\mathbf{C} \mid t) p_{T}(t) d t ; \quad \mathbf{C} \mid T \sim \mathcal{W}_{d}^{\mathbb{C}}(L, \boldsymbol{\Sigma})$.

The resulting distribution is known as the K-Wishart distribution [1] and in closed form is:

$$
\begin{aligned}
p_{C}(\mathbf{C})= & \mathrm{KW}(\mathbf{C} ; L, \alpha, \boldsymbol{\Sigma}) \\
= & \frac{2|\mathbf{C}|^{L-d}}{\mathrm{I}(L, d) \Gamma(\alpha)|\boldsymbol{\Sigma}|^{L}}(L \alpha)^{\frac{\alpha+L d}{2}}\left(\operatorname{tr}\left(\boldsymbol{\Sigma}^{-1} \mathbf{C}\right)\right)^{\frac{\alpha-L d}{2}} \\
& \times \mathrm{K}_{\alpha-L d}\left(2 \sqrt{\operatorname{L\alpha tr}\left(\boldsymbol{\Sigma}^{-1} \mathbf{C}\right)}\right)
\end{aligned}
$$

where $\operatorname{tr}(\cdot)$ and $|\cdot|$ denote the trace operation and determinant, respectively, $I(L, d)$ is a normalization constant, and $K_{n}(\cdot)$ is the modified Bessel function of the second kind with order $n$. Note that the PDF is parametrized by the shape parameter $\alpha$, the number of looks $L$ and the scale matrix $\boldsymbol{\Sigma}$.

Instead of calculating Maximum Likelihood (ML) estimates of the gamma distribution texture parameter, the method of matrix log-cumulants (MoMLC) is adopted, which have been proved to be a feasible and effective estimation technique and relies on the properties of the Mellin transform. The matrix log-cumulant equations of the K-Wishart distribution are [9]:

$$
\kappa_{1}(\mathbf{C})=\ln |\boldsymbol{\Sigma}|+\psi_{d}^{0}(L)+d\left(\psi^{0}(\alpha)-\ln (\alpha L)\right)
$$

$\kappa_{\nu}(\mathbf{C})=\psi_{d}^{\nu-1}(L)+d^{\nu} \psi^{\nu-1}(\alpha), \quad \nu>1$

where $\nu$ is the matrix log-cumulant order, whereas $\psi_{d}^{\nu}(\cdot)$ and $\psi^{\nu}(\cdot)$ are the multivariate and the ordinary polygamma function, respectively [9].

\section{MARKOVIAN FUSION APPROACH}

In order to include the contextual information disregarded by the pixel-wise EM technique and to gain robustness against speckle, we adopt an MRF model which generally allows contextual and further information sources to be integrated in Bayesian image processing. This is done by a MAP decision rule as the minimization of suitable energy functions [5]. As a consequence, an MRF can be applied to model the prior distribution of the class labels, and a mixture of K-Wishart distributions is used to model the class-conditional PDF for the PolSAR data statistics.

\subsection{Markov Random Field model}

Let $S=\{s=(i, j) ; 1 \leq i \leq M, 1 \leq j \leq N\}$ and $K$ denote the pixel lattice and the set of all possible labels in the clustering map, respectively. Therefore, a label random field $\Omega=\left\{\omega_{s}, \omega_{s} \in K, s \in S\right\}$ defined on the $S$ can be treated as an MRF with a given neighborhood system if the Markovian property holds for each site $s$ [8]. We introduce an isotropic second-order neighborhood system and the related set of pairwise cliques. For each site, the neighbors are the eight surrounding pixels [4]. According to the Hammersley-Clifford theorem, the joint probability distribution of a Markov field is a Gibbs distribution [8]. Therefore, the PDF of $\Omega$ has the form

$P_{\Omega}(\omega)=Z^{-1} \exp [-U(\omega)]=Z^{-1} \exp \left[-\sum_{c} V_{c}\left(\omega_{c}\right)\right]$

where $\mathrm{U}(\omega)$ and $V_{c}\left(\omega_{c}\right)$ are called Markov energy function of class labels and potential function, respectively; $\mathrm{Z}$ is the normalizing constant; and c indicates a clique of a neighborhood system. Consequently, local spatial correlations in $\Omega$ can be modeled by defining suitable potential functions $V_{c}$. According to the assumption of isotropy, there is a single MRF model parameter which is known as the spatial interaction parameter of the pairwise cliques and the potential can therefore be simplified to $[4,8]$

$$
V_{c}\left(\omega_{s}, \omega_{N(s)}\right)=\left\{\begin{array}{rc}
-\beta & \text { if } \omega_{s}=\omega_{N(s)} \\
0 & \text { otherwise }
\end{array}\right.
$$

where $N(s)$ is the neighborhood of the $s \in S$.

\section{K-WISHART MRF CLASSIFIER}

Image segmentation involves observed data and unobserved data to be recovered. In the case of hidden MRFs, the unobserved class labels $\omega$ are modeled as an MRF, as defined in (7) with the energy function $U$ depending on a parameter $\beta$ [8]. The observed MLC image can therefore 
be described by a mixture of K-Wishart distributions and are assumed to be conditionally independent given $\omega$ :

$$
P_{C \mid \Omega}(\mathbf{C} \mid \omega ; \theta)=\exp \left[\sum_{s \in S} \log \mathrm{KW}\left(\mathbf{C}_{s} \mid \omega_{s} ; \alpha, \boldsymbol{\Sigma}\right)\right]
$$

In order to develop the K-Wishart MRF classifier using the MAP criterion, we need to obtain the conditional probability mass function (PMF) of the label random field $\omega$ given the observed field $\mathbf{C}$, in terms of the Bayesian rule, which can be formulated as

$$
P_{\Omega \mid \mathbf{C}}(\omega \mid \mathbf{C} ; \theta, \beta) \propto P_{C \mid \Omega}(\mathbf{C} \mid \omega ; \theta) P_{\Omega}(\omega \mid \beta),
$$

where $\theta=\{\alpha, \Sigma\}$ is defined as the vecor of the parameters of the K-Wishart distribution; $P_{\Omega}$ and $P_{\Omega \mid \mathbf{C}}$ are the prior $\mathrm{PMF}$ of the class labels and the posterior probability of class membership, i.e., the probability that $\mathbf{C}$ belongs to $\omega$ given the observation $\mathbf{C}$, respectively. To model the joint prior probability $P_{\Omega}$, an MRF model is applied. Using the pseudo-likelihood defined in [8], the complete likelihood is given by

$$
P(\mathbf{C}, \omega \mid \theta, \beta) \approx \prod_{s \in S} \mathrm{KW}\left(\mathbf{C}_{s} \mid \omega_{s} ; \theta\right) P_{\Omega}\left(\omega_{s} \mid \omega_{N(s)} ; \beta\right)
$$

Therefore, the conditional field $\omega$ given $\mathbf{C}$ is a Markov field (just as $\omega$ ) and its posterior energy is

$$
U(\omega \mid \mathbf{C} ; \theta, \beta)=U(\omega \mid \beta)-\sum_{s \in S} \log \mathrm{KW}\left(\mathbf{C}_{s} \mid \omega_{s} ; \theta\right)
$$

Thus, the MAP estimate which maximize the posterior probability $P(\omega \mid \mathbf{C} ; \theta, \beta)$ is equivalent to minimizing the posterior energy. The K-Wishart MRF clustering algorithm consists of the following processing stages:

(1) Non-contextual step: this step generates an initial noncontextual clustering map by applying the unsupervised $\mathrm{K}$-Wishart classifier. Unsupervised segmentation of $\mathrm{C}$ matrices is achieved using an expectation-maximization (EM) approach. The standard EM-algorithm consists of an E-step that estimates class likelihoods using KWishart distribution and an M-step that updates all class parameters [7]. The final class partition has $k$ classes with associated K-Wishart parameters for each class.

(2) MRF step: Under the assumption of the independency of the covariance matrices and finite mixture of KWishart distributions in the PolSAR image, the EM algorithm is applied to the described MRF model. The modefield formulation of EM is used, that represents an approximation in the related likelihood function and makes EM computationally affordable also in the application to contextual models [8]. At the convergence of the EM, the final segmentation is obtained.

\section{EXPERIMENTAL RESULTS}

The non-Gaussian K-Wishart classifier was adopted for a test image with eight classes that are generated with eight-look, dual-pol K-Wishart distributed data. The polarimetric matrix and texture parameters are taken from a real data-set to simulate classes with properties of the real image. To initialize the expectation maximization process, each observation is assigned randomly to one of eight classes. The K-Wishart PDF is dependent on the number of looks $L$ which are assumed to be independent. Because of existing some correlation between image pixels in real data, actual number of correlated looks need to be substituted with effective number of independent looks (ENL) to fit the data. We incorporate ENL estimation in the EM algorithm [10]. The K-Wishart MAP classifier already provides good classification performances in the non-contextual step, with accuracies higher than $75 \%$ for all the classes and overall accuracy of $91 \%$. Even though the considered operational setting is unsupervised, such a quantitative accuracy analysis is feasible for this dataset, since the true label of each pixel is known by definition. As expected, classification results appear noisy due to insufficient speckle filtering, but the contextual KWishart MRF classifier further improved the result, yielding a $8 \%$ increase in the overall accuracy and accuracies higher than $98 \%$ for all the classes. The classification result can be seen for the simulated data in Fig. 1. As a second experiment, the image of an agricultural area from an airborne EMISAR, L-band, quad-pol SAR flight over Foulum Denmark in 1998 is tested. The appropriate number of classes for this real dat-set is automatically determined by goodness-of-fit testing of each class model to the data samples [10] and this gives a different number of classes depending on the constraints of the chosen model. The standard-Wishart classifier was adopted for the image and Fig. 2 shows the result and improvement of the clustering by standard-Wishart MRF classifier. For this textured image, standard-Wishart is not a good selection and K-Wishart can better model the data. So K-Wishart classifier was chosen and Fig. 3 shows the classification result. As it is seen from Fig. 2 and Fig. 3 , the standard-Wishart classifiers gives us 34 classes, whereas K-Wishart classifier found 18 classes for this data. Since the standard-Wishart has difficulty in grouping the highly non-Gaussian classes into single groups, it fits more Gaussian-constrained distributions to the nonGaussian data classes. A relevant improvement in spatial regularity, accuracy and a strong smoothing of homogeneous areas is visually evident when incorporating MRFs, while class boundaries and point targets are preserved in the contextual clustering.

\section{CONCLUSIONS}

In this paper, a novel unsupervised clustering algorithm for PolSAR imagery has been developed by combining the MRF approach to Baysian image classifier and a finite mixture technique for PDF estimation, which is designed to yield homogeneous classification results. We tested the proposed algorithm over two examples and showed the classification accuracies before and after applying MRFs. The results shows improvement on segmentation of pixel-wise K-Wishart clustering. The accuracy of the proposed algorithm was validated on the simulated 


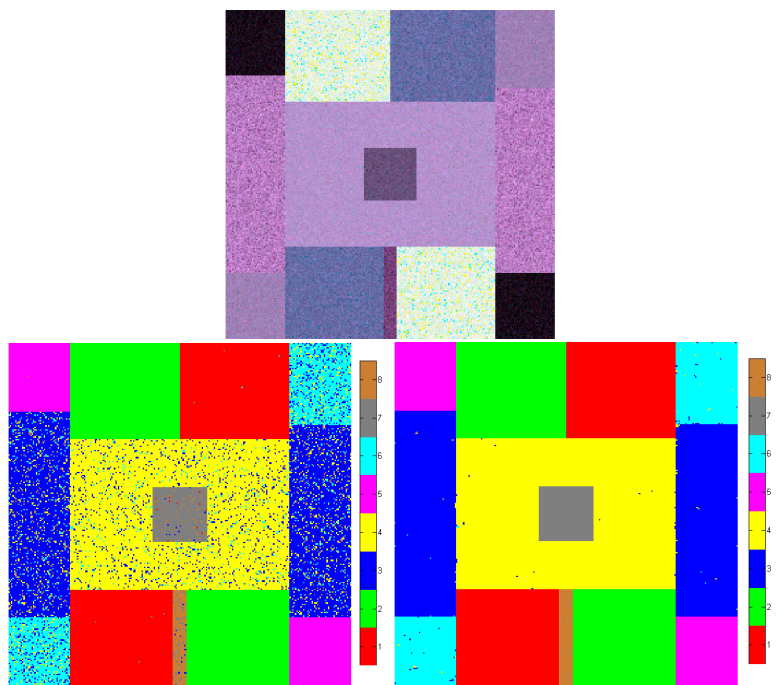

Figure 1. Quasi-Pauli (dual-pol) RGB image (top) and non-contextual (left bottom) and contextual (right bottom) $K$-Wishart clustering of simulated test pattern.

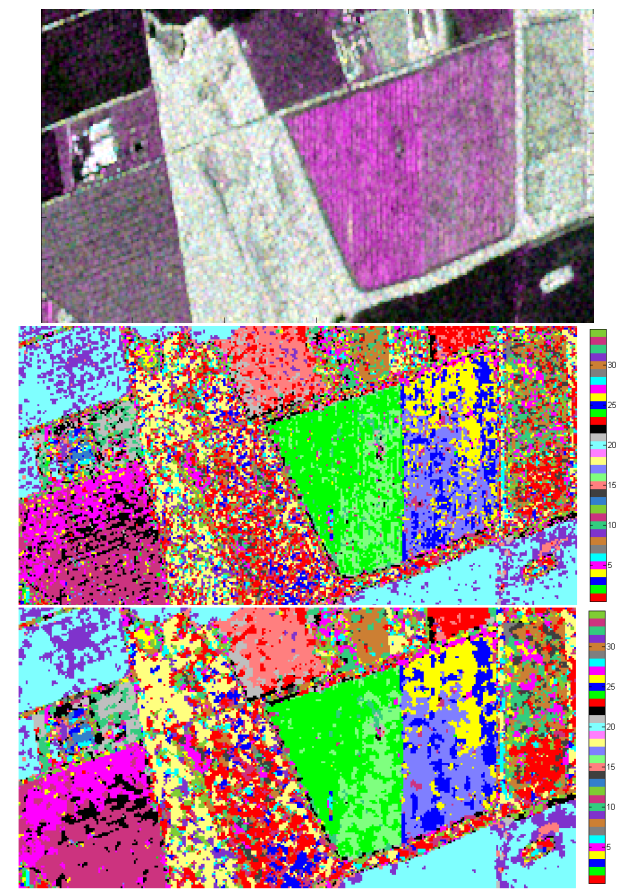

Figure 2. Pauli RGB image (top) and standard Wishart non-contextual (middle) and contextual clustering of Foulum dataset, eight-looks, 34 classes found.

test pattern and quad-pol SAR images of Foulum Denmark. In both cases, the effectiveness of MRF models in improving the accuracy of PolSAR image clustering has been experimentally remarked. Future developments of this study will include extending the MRF modeling to change detection applications with PolSAR data.

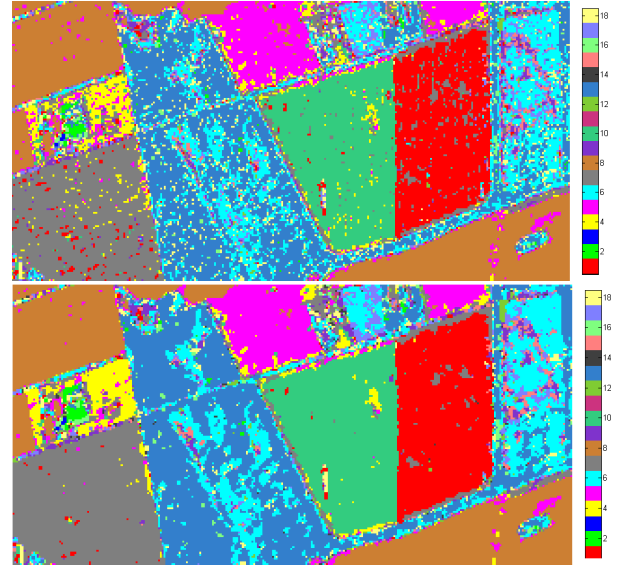

Figure 3. Non-contextual (top) and contextual (bottom) $K$-Wishart clustering of Foulum dataset, 18 classes found.

\section{REFERENCES}

[1] A. Doulgeris, S. Anfinsen, and T. Eltoft, "Classification with a non-Gaussian model for PolSAR data," IEEE Trans. Geosci. Remote Sensing, vol. 46, no. 10, pp 2999-3009, Oct. 2008.

[2] C. C. Freitas, A. C. Frery, and A. H. Correia, "The polarimetric $\mathrm{G}$ distribution for SAR data analysis," Environmetrics, vol. 16, pp. 13-31, 2005.

[3] J. Lee, M. Grunes, and R. Kwok, Classification of multi-look polarimetric SAR imagery based on the complex Wishart distribution, Int. J. Remote Sensing, vol. 15, 111994.

[4] S. Li, "Markov random field modeling in image analysis", Springer, 2009.

[5] S. B. Serpico, G. Moser, "Weight parameter optimization by the Ho-Kashyap algorithm in MRF models for supervised image classification", IEEE Trans. Geosci. Remote Sensing, vol. 44, no.12, pp. 3695-3705, 2006.

[6] G. Moser, S. B. Serpico, 'Unsupervised change detection from multichannel SAR data by Markovian data fusion", IEEE Trans. Geosci. Remote Sensing, vol. 47, no. 7, pp. 2114-2128, 2009.

[7] A. P. Dempster, N. M. Laird, and D. B. Rubin, "Maximum likelihood from incomplete data via teh EM algorithm," Journal of the Royal Statistical Society. Series B, vol. 39, no. 1, pp. 1-38, 1977.

[8] G., Celeux, F. Forbes and N. Peyrand, "EM procedures using mean field-like approximations for Markov model-based image segmentation," Pattern Recognition, vol. 36, no. 1, pp. 131-144, 2003.

[9] S. N. Anfinsen and T. Eltoft, "Application of the matrix-variate Mellin transform to analysis of polarimetric radar images," IEEE Trans. Geosci. Remote Sensing, vol. 49, no. 7, 15 pp., July, 2011.

[10] A. Doulgeris, S. Anfinsen, and T. Eltoft, "Automated Non-Gaussian Clustering of Polarimetric Synthetic Aperture Radar Images," IEEE Trans. Geosci. Remote Sensing, vol. 49, no. 8, To appear in 2011. 